\title{
Definition and Properties of Ideal Amorphous Solids
}

\author{
Zbigniew H. Stachurski \\ Department of Engineering, Faculty of Engineering and Information Technology, The Australian National University, \\ Canberra, ACT 0200, Australia
}

(Received 12 August 2002; published 15 April 2003)

\begin{abstract}
It is proposed that two ideal amorphous structures, type I and type II, based on maximally random jammed packing of spheres of equal size, form a distinct class of ideal amorphous solids. The ideal amorphous structures contain wide variations in local density, limited by the condition of solidity. Four distinct characteristics, based on statistical geometry and topology, are shown to define this class. Voronoi tessellations carried out on simulated cells of random packed spheres and amorphous polymers give a broad distribution of individual volumes, skewed, with a tail at the high volume end.
\end{abstract}

DOI: $10.1103 /$ PhysRevLett.90.155502

Torquato et al. [1] pointed out the futility of searching for the universal density of a randomly close packed (RCP) body and, instead, proposed the existence of a maximally random jammed (MRJ) state, encompassing a group of structures with maximized disorder parameters.

This view opens the way for the definition of ideal amorphous solids (IAS), which can serve as models of real materials, including amorphous polymers. The search for a definition of an ideal amorphous solid stems from the author's interest in a complete theory of plasticity in amorphous solid polymers. It derives from the understanding of, and analogy with, crystalline solids, for which the dislocation theory of plasticity has been so successful. The development of plasticity theory in crystalline solids depended on the confluence of three essential elements: (i) theory of crystallography providing the definition of ideal crystalline solids, (ii) mechanisms of plastic deformation (edge and screw dislocations), and (iii) an experimental method of verification (electron microscopy).

The above three elements are also necessary and essential for a complete understanding of plasticity in amorphous polymers. A method equivalent to electron microscopy is effectively provided by computer simulations and nuclear magnetic resonance $[2,3]$. Many theories of mechanisms of plastic deformation in polymers exist in published literature [4]. However, the first essential element, namely, a theory of the structure of amorphous solid polymers, is not developed yet in sufficient detail to afford the clarity of understanding required to describe precisely individual molecular motions and the accompanying changes in nanostructure during plastic deformation occurring in amorphous polymers. It is frequently tacitly assumed that amorphous materials are found at the limits of disorder, and especially for polymers, amorphicity is usually defined by what it is not, rather than by what it is.

From the work of Torquato et al., the essential points of direct relevance to amorphous materials can be summarized as follows:
PACS numbers: 61.43.-j, 03.65.Vf, 36.40.-c, 62.25.+g

(i) The "universal" density of RCP structure cannot be defined mathematically, nor can it be found experimentally -it does not exist as a unique quantity. This is inferred from the conflict between "random" and "close packed" states, since arbitrarily small increases in disorder can be achieved at the expense of small decreases in density, and vice versa.

(ii) Introduction of the concept of the MRJ state in which all particles (spheres) are jammed; a jammed particle cannot be translated. The definition of the MRJ state is based on minimization of order parameters ( $Q$ or $T$ ), subject to the constraint of the jammed state.

(iii) A small number of "rattler" particles is admitted; therefore, a variety of structures must exist, bound by density and randomness limits.

The intention here is to introduce a more narrowly defined MRJ structure as an IAS; therefore the issue of density must be elaborated on. Consider a body created by random dense packing of $N$ hard spheres of identical diameter, $a$, which are to a large extent in contact. The body is not subject to any force field (including gravity). The spheres do not overlap, nor is there any attraction or friction between them; therefore there is no preference for the number of nearest neighbors other than the requirement for dense packing.

The body has numerical density, $\rho_{b}=N / V$, which is conveniently expressed in terms of a packing fraction. Let the body be divided into smaller pieces, and the pieces divided into smaller pieces. For many subsequent divisions the density of each piece will be the same. Eventually, there will be a piece of the smallest representative volume element (RVE) for which the density is still the same and equal to $\rho_{b}$ (this translational invariance in the mean is a requirement imposed on all macroscopically large disordered systems $[5,6])$. By implication, a further division into volumes smaller than RVE will result in variations of density. Therefore, $\rho_{b}$ is an average density of the body: $\rho_{b}=\bar{\rho}$. In the limit, the largest variations in local densities, $\rho_{i}$, will be found when volumes around each sphere are expressed in terms of corresponding Voronoi polyhedra [7,8]. From the implicit 
assumptions, one can make the following observations: (1) Density fluctuations for individual spheres are in both positive and negative sense from the average density. (2) There exists a well defined maximum density, $\rho_{\max }=$ $\pi / \sqrt{18} \approx 0.74$, that occurs in densely packed structures [2] and provides the absolute limiting value on the righthand side of the distribution. (3) For a symmetric distribution around an asymptotic average value, $\bar{\rho}=0.64$ [1], the following is true $\left|\rho_{\min }-\bar{\rho}\right|=\left|\rho_{\max }-\bar{\rho}\right|$, providing a value for the minimum density, $\rho_{\min } \approx 0.54$ [curve $(a)$ in Fig. 1]. (4) For a nonsymmetric distribution [curve (b) in Fig. 1] the minimum value of density (for any sphere inside the body) is less than that for the symmetric case, but greater than zero: $0 \ll \rho_{\min }<0.54$.

An MRJ state implies as wide a distribution of local densities as possible, whereas solidity requires dense packing and imposes spatial constraints on the spheres. Rotations of spheres can be omitted, but translations must be considered. A sphere is in a fixed position (called "jammed" in [1]) if it is supported on its surface by a
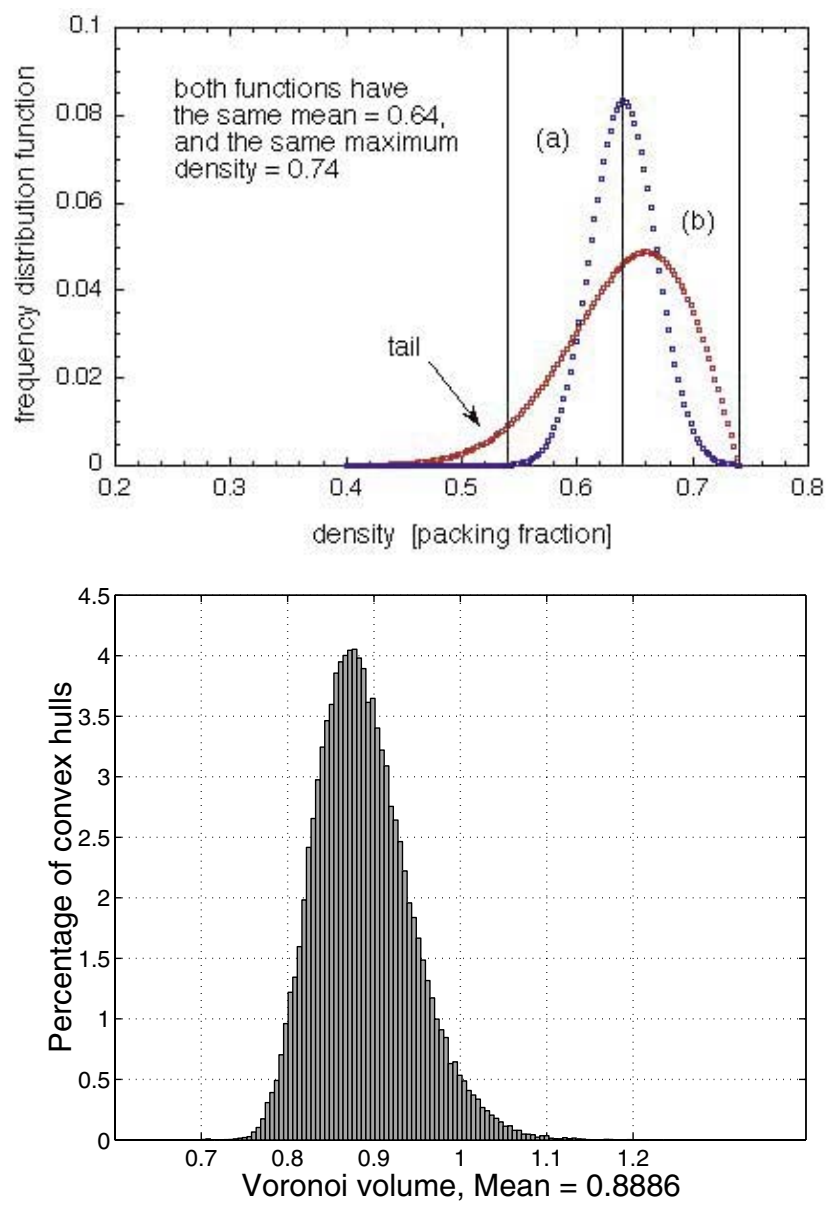

FIG. 1 (color online). Top: hypothetical frequency density distribution functions, (a) symmetric, and (b) skewed. Bottom: Voronoi volume distribution for random packing of spheres with an average packing fraction $=0.59$. Notice the tail at high volumes. minimum of four points with at least one of these on the other hemisphere, which is equivalent to saying that its center is located within the tetrahedron formed on the four points. If all spheres are in fixed positions, then the body is perfectly solid. However, the body can remain solid and stable even if a number of spheres inside it are allowed some degree of kinetic freedom (called "rattlers" in [1]).

We conclude that the sphere's position is (i) fixed, when for $4 \leq N_{C} \leq 9$ touching contacts with neighboring spheres, no more than $\left(N_{C}-1\right)$ are located on one hemisphere; for $N_{C}>9$ the sphere is always in a fixed position (the neighboring spheres must also be in fixed positions) and (ii) loose, when the touching contacts, $2 \leq N_{C} \leq 9$ in number, are all on one hemisphere only; the sphere is contained but not fixed and can move to other loose positions (the restriction on neighboring spheres being in fixed positions need not apply).

It is postulated that, for every $V>\operatorname{RVE}$, a necessary structural solidity condition can be expressed as follows:

$$
J_{S}=\frac{1}{N} \sum_{i=1}^{N} J_{i} \leq J_{\text {crit }},
$$

where $J_{i}=\sqrt{u^{2}+v^{2}+w^{2}}, u, v$, and $w$ are the maximum allowed displacements of a loose sphere in the $x, y$, and $z$ directions, respectively, and $0 \leq J_{\text {crit }}$ is a characteristic property for a given type of structure. The value of $J_{\text {crit }}$, or rather the distribution of $J_{i}^{\prime}$ s must be so chosen that for any given RVE the body remains solid. The size of RVE is defined by the mathematical probability statement

$$
P(|\bar{\mu}-\bar{\rho}| \leq d) \geq \alpha,
$$

where $\mu$ is the mean density of RVE, $\bar{\rho}$ is the mean density of the body, and the tolerance, $d$, and the confidence limit, $\alpha$, are chosen in accordance with specific requirements. With this preamble, it is now possible to put forward the definition of an ideal amorphous solid of type I and type II:

Definition 1: An MRJ structure, composed of identical size spheres, is an ideal amorphous structure of type I (IAS-I) if it possesses all the characteristics described above, and for which the RVE attains a maximum size subject to conditions (1) and (2).

On a macroscale, such a body is said to have an amorphous structure without similarity and/or translational order. An arbitrary rotation about any point within the body results in the same density distribution, and hence the same physical properties. The structure is motion invariant and isotropic [5,6].

On a nanoscale, the IAS possesses four statistical geometry elements:

1. A translational element is defined by a random walk along centers of spheres in contact with each other. Let the initial position of the walk be denoted as $\mathbf{R}_{0}$, and subsequent steps by $\mathbf{r}_{1}, \mathbf{r}_{2}, \ldots, \mathbf{r}_{n}$; then the final position is 


$$
\mathbf{R}_{n}=\mathbf{R}_{0}+\sum_{i=1}^{n} \mathbf{r}_{i}
$$

If the steps are random and mutually independent, then the mean-square-root end-to-end distance of the walk is a measure of this element, and it must obey the relationship

$$
\left\langle R_{n}^{2}\right\rangle^{(1 / 2)}=a \sqrt{n},
$$

where $n$ is the number of steps taken and $\left\langle R_{n}^{2}\right\rangle / n$ is constant. For a self-avoiding random walk (particularly relevant to Definition 2 below) the mean-square-root endto-end distance of the walk is [9]

$$
\left\langle R_{n}^{2}\right\rangle^{(1 / 2)}=\sqrt{A n^{(6 / 5)}},
$$

where $A$ is a constant and $\left\langle R_{n}^{2}\right\rangle / n$ diverges as $n \rightarrow \infty$.

2. A spherical element defines the disposition of the contact points on the sphere's surface. Its measure must reflect the departure from symmetrical arrangement of the contacts. The $Q$ measure used by Toquato et al. [1] is suitable. An alternative measure is proposed below.

Let the contact points of a chosen sphere be numbered sequentially, denoted by the index $k$. The contact angle, $\beta_{k l}$, is defined as an angle formed by two lines emanating from the center of the sphere to the two contact points, $k$ and $l$, where $l=k+1$. Then the contact angle variation for an individual sphere, $s_{\beta}$, is defined by the following expression:

$$
s_{\beta}=\frac{1}{N_{C}} \sum_{k=1}^{N_{C}-1}\left|\beta_{k l}-\beta_{(k+1)(l+1)}\right|,
$$

with $l$ taking on the value of 1 when $l>N_{C}-1$. It is noted that $s_{\beta}$ reaches maximum value when the greatest irregularity in the distribution of the contact points on the sphere's surface is found and tends to the value of zero when the spatial disposition of contacts becomes symmetrical (for any allowed value of $N_{C}$ ).

3. A mirror element is defined by the requirement that at every contact point, the touching spheres are a mirror image of each other. This element ensures that the spheres are identical in size and type. In an IAS-I, all spheres possess a minimum of 2 and a maximum of 12 mirror elements.

4. A central element describes a geometrical relationship between a sphere and its corresponding Voronoi polyhedron. The sphere possesses a central element if it is in the center of its Voronoi polyhedron. This occurs only and if the surrounding neighbors are in centrosymmetric positions with respect to the sphere.

Definition 2: An ideal amorphous structure of type II, composed of linear freely jointed chains of hard spheres, is one in which the positions in space of the centers of spheres in the chains are identical with those in IAS-I.

The above structure can be constructed along the following lines: Consider a cell containing IAS-I with
$N$ randomly packed equal sized spheres (where $N$ is a large number). Each sphere has a number of touching neighbors. Make a chain of touching spheres: Chose one sphere as the starting point and thread it with one of its touching neighbors. Next, thread the second sphere at random with one of its touching neighbors, except the one already joined to. Continue in this manner the three dimensional self-avoiding walk (SAW) until a chain of $n-1$ links is made. Next, choose another unattached starting sphere and, following the above process, construct another chain of $n$ spheres. Repeat the process until $(N / n)$ chains are formed. Since $N \gg n$, there are many ways to form the chains, and therefore there exists a finite probability that it is possible to form $(N / n)$ chains without violating the SAW, and without any "free" sphere(s) left over. This cell of linear freely jointed chains constitutes an ideal polymeric amorphous solid.
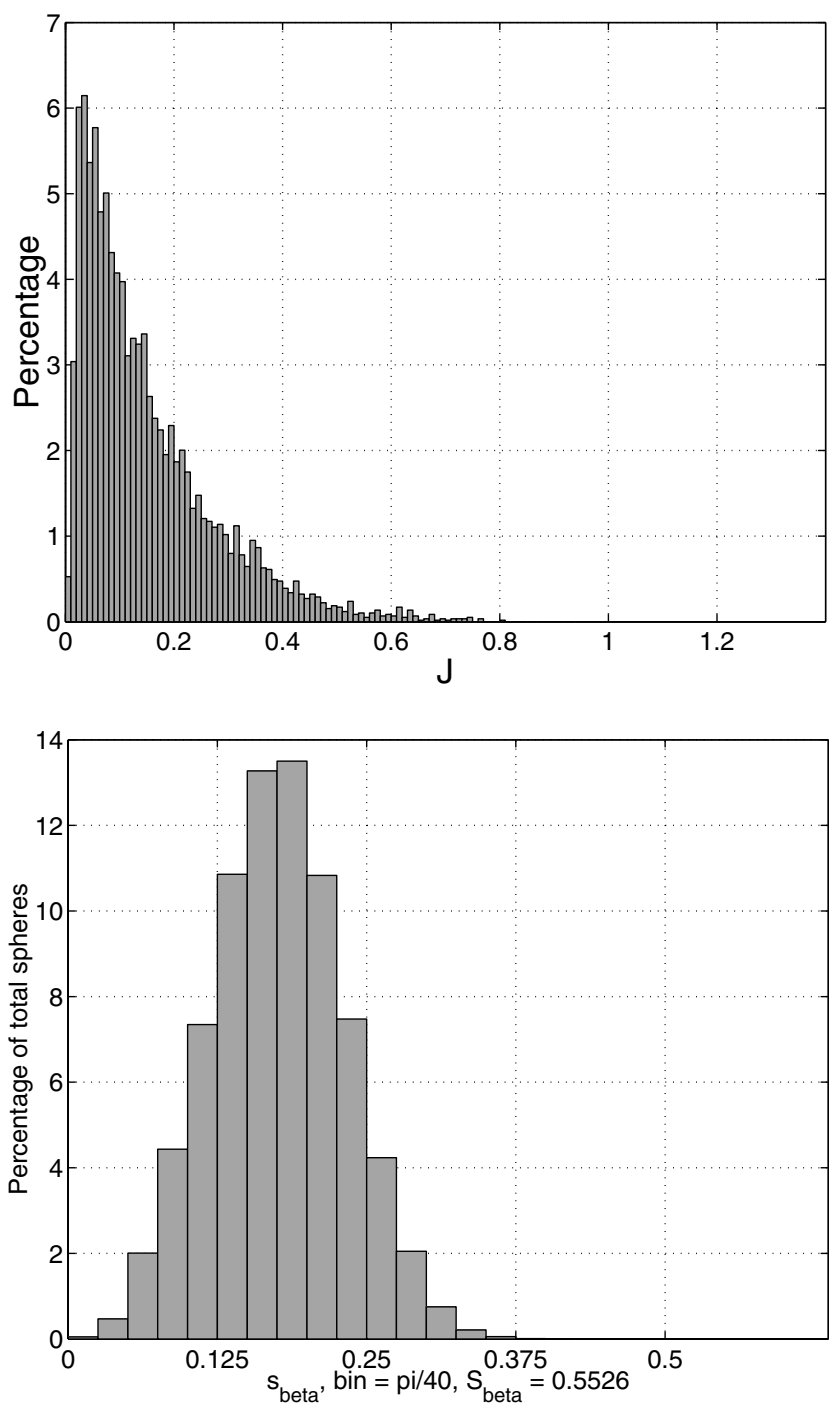

FIG. 2. Top: distribution of $J_{i}^{\prime} s$ [see Eq. (1)], $J=1$ corresponds to one sphere diameter. Bottom: corresponding distribution of $s_{\beta}^{\prime} s$ in radians [see Eq. (6)]. 

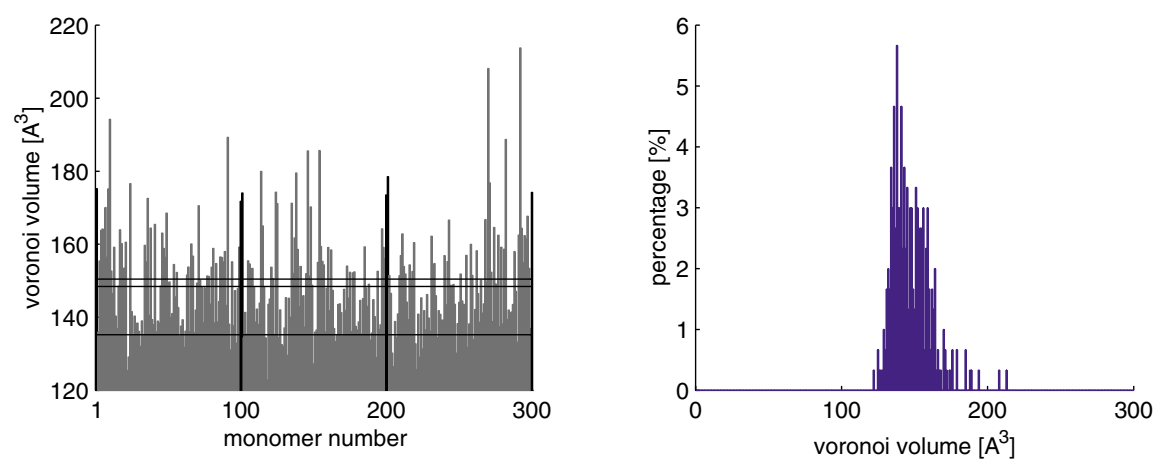

FIG. 3 (color online). Left: Voronoi volumes for a sequence of monomers on a chain in PMMA. Right: distribution of the Voronoi volumes from the graph on the left.

By virtue of the fact that the starting structure is an IAS of type I, and since the arrangement of the spheres is not altered or disturbed by the process of chain formation, the ideal amorphous solids of type I and type II have the same geometrical properties and therefore must belong to the same class of amorphous structures. Close packed spheres with a preference for $n$ contact points [10], or two or three different kinds, also form disordered structures [2], as do spheres each of different size [11]. It is anticipated that they will form separate amorphous classes.

MRJ cells of densely packed spheres have been computer simulated using statistical geometry algorithm and Voronoi tessellations were carried out [12]. An example of the distribution of Voronoi volumes for a cell of packing fraction $=0.59$ and $6.6 \%$ loose spheres is shown on the bottom in Fig. 1. The result supports the nonsymmetrical distribution shown on the top. Figure 2 shows the corresponding distribution of $J_{i}$, Eq. (1), and the corresponding distribution of $s_{\beta}$, Eq. (6). The data support the conjecture of a random body. Also, amorphous cells of poly (methyl methacrylate) (PMMA) and poly (carbonate) were simulated using standard methods [13]. Measurements of Voronoi volumes, $\left(V_{V}\right)$, for all individual atoms were carried out. The Voronoi volumes of atoms belonging to the same monomer were added together, the result showing scattered values as can be seen on the left in Fig. 3. The corresponding frequency distribution of the Voronoi volume has the characteristic skewed shape, as shown on the right. The example included here is one of many that we have obtained for individual or groups of atoms: (i) for united atom freely jointed chains [14] and (ii) for chiral $\mathrm{CH}_{3}$ groups in polypropylene [15]. No symmetrical distributions have been published, although suggestions have been made [16].

The significance of the contribution is in two areas: (i) emerging structure of a theory of ideal amorphous solids. The four statistical geometry elements define precisely and adequately the properties of IAS type I and type II. Elaboration will be given in a full paper [12]. (ii) The understanding of the wide density fluctuations in amorphous polymers is helpful in developing a theory of plasticity in glassy polymers, based on the concept of constriction points published before [17,18].

[1] S. Torquato, T. M. Truskett, and P. G. Debenedetti, Phys. Rev. Lett. 84, 2064 (2000).

[2] J. Villain, K. Y. Szeto, B. Minchau, and W. Renz, in Computer Simulation Studies in Condensed Matter Physics III, edited by D. P. Landau, K. K. Mon, and H.-B. Schütelr (Springer-Verlag, Berlin, Heidelberg, 1991).

[3] D. N. Theodorou and U.W. Suter, Macromolecules 18, 1467 (1985).

[4] Z. H. Stachurski, Prog. Polym. Sci. 22, 407 (1997).

[5] J. M. Ziman, Models of Disorder (Cambridge University Press, Cambridge, 1979).

[6] I. M. Lifshits, S. A. Gredeskul, and L. A. Pastur, Introduction to the Theory of Disordered Systems (John Wiley \& Sons, New York, 1988).

[7] G. Voronoi and J. Reiner, Angew. Math. 134, 198 (1908).

[8] W. Brostow, M. Chybicki, R. Laskowski, and J. Rybicki, Phys. Rev. B 57, 13448 (1998).

[9] M. N. Barber and B.W. Ninham, Random and Restricted Walks (Gordon and Breach, New York, 1970).

[10] K. Tsumuraya, K. Ishibashi, and K. Kusunoki, Phys. Rev. B 47, 8552 (1993).

[11] D. L. Weaire and S. Hutzler, The Physics of Foams (Clarendon Press, Oxford, New York, 1999).

[12] L. T. To, Z. H. Stachurski, and T. R. Welbery (to be published), preprint available on request.

[13] L. A. Pfister and Z. H. Stachurski, Polymer 43, 7417 (2002).

[14] D. Rigby and R. J. Roe, Macromolecules 23, 5312 (1990).

[15] M. F. Sylvester, S. Yip, and A. S. Argon, Computer Simulation of Polymers, edited by R. J. Roe (PrenticeHall, Englewood Cliffs, NJ, 1991), Chap. 8.

[16] G. Floudas, T. Pakula, M. Stamm, and E.W. Fischer, Macromolecules 26, 1671 (1993).

[17] Z. H. Stachurski, J. Mater. Sci. 21, 3237 (1986).

[18] S. F. Edwards and Th. Vilgis, Polymer 28, 375 (1987). 